\title{
O processo de envelhecimento na sociedade: uma análise da literatura com foco na autopercepção dos idosos e na enfermagem
}

\author{
The aging process in society: an analysis of the literature focusing on the self-perception \\ of the elderly and on nursing \\ El proceso de envejecimiento en la sociedad: un análisis de la literatura con foco en la \\ autopercepción del anciano y en la enfermería
}

Adilson Mendes de Figueiredo Júnior ${ }^{1 *}$, Yasmin Martins de Sousa1, Christian Boaventura dos Santos $^{2}$, Maria dos Remédios Tavares², Raquel Ferreira da Silva ${ }^{3}$, Renan Barbosa Fortuna da Silva ${ }^{4}$, Deborah Pereira Reis ${ }^{4}$, Eliane Conceição da Costa ${ }^{4}$, Luccas Eduardo Barros Siqueira ${ }^{4}$, Maira Lohana Carvalho Guedes ${ }^{4}$.

\section{RESUMO}

Objetivo: Analisar como os idosos estão vivenciando o processo de envelhecimento na sociedade. Métodos: $O$ estudo se deu a partir da pesquisa qualitativa tendo ênfase na pesquisa bibliográfica, para isso, procurou entender por meio da literatura de que maneira os idosos vivenciam o processo de envelhecimento e até que ponto tal procedimento interfere em suas vidas. Resultados: Os resultados obtidos demonstram que 0 processo de envelhecimento não é algo homogêneo e possui diversas particularidades, onde o mesmo é vivenciado de maneira única por cada indivíduo, demonstrando que o mesmo não está pautado apenas nas características do corpo, mas em sua capacidade cognitiva e disposição física e motora, dentre outras. Considerações finais: Cabe a enfermagem, Em suas funções educativas e políticas, compromete-se com os direitos sociais e de saúde do idoso, defendendo sua dignidade, estimulando o senso de participação do idoso, visando a integração com as pessoas, trabalhando a particularidade e singularidade do idoso, enquanto ao mesmo tempo Considerando que fazem parte de um todo complexo e contraditório, promovendo assim a saúde desses públicos-alvo.

Palavras-chave: Envelhecimento, Enfermagem, Saúde.

\begin{abstract}
Objective: To analyze how the elderly are experiencing the aging process in society. Methods: The study was based on qualitative research, with an emphasis on bibliographic research, for that, it sought to understand through the literature how the elderly experience the aging process and to what extent this procedure interferes in their lives. Results: The results obtained demonstrate that the aging process is not homogeneous and has several particularities, where it is experienced in a unique way by each individual, demonstrating that it is not based only on the characteristics of the body, but on its cognitive capacity. and physical and motor disposition, among others. Final considerations: It is up to nursing, In its educational and political functions, it is committed to the social and health rights of the elderly, defending their dignity, stimulating the elderly's sense of participation, aiming at integration with people, working on the particularity and singularity of the elderly, while at the same time considering that they are part of a complex and contradictory whole, thus promoting the health of these target audiences.
\end{abstract}

Key words: Aging, Nursing, Health.

\footnotetext{
1 Universidade do Estado do Pará (UEPA), Belém - PA. *E-mail: adilsonmdfj@hotmail.com

2 Universidade Federal do Pará (UFPA), Belém - PA.

${ }^{3}$ Universidade da Amazônia (UNAMA), Belém - PA.

${ }^{4}$ Escola Superior da Amazônia (ESAMAZ), Belém - PA.
} 


\section{RESUMEN}

Objetivo: Analizar cómo los ancianos están viviendo el proceso de envejecimiento en la sociedad. Métodos: El estudio se basó en una investigación cualitativa, con énfasis en la investigación bibliográfica, para eso, buscó comprender a través de la literatura cómo los ancianos experimentan el proceso de envejecimiento y en qué medida ese procedimiento interfiere en sus vidas. Resultados: Los resultados obtenidos demuestran que el proceso de envejecimiento no es homogéneo y tiene varias particularidades, donde es vivido de manera única por cada individuo, demostrando que no se basa solo en las características del cuerpo, sino en su capacidad cognitiva. y disposición física y motora, entre otros. Consideraciones finales: Corresponde a la enfermería, En sus funciones educativas y políticas, está comprometida con los derechos sociales y de salud de las personas mayores, defendiendo su dignidad, estimulando el sentido de participación de las personas mayores, visando la integración con las personas, trabajando la particularidad y singularidad de las personas mayores, considerando al mismo tiempo que forman parte de un todo complejo y contradictorio, favoreciendo así la salud de estos públicos objetivo.

Palabras clave: Envejecimiento, Enfermería, Salud.

\section{INTRODUÇÃO}

O processo de envelhecimento está associado por alterações ligadas a fatores biológicos, psicológicos e sociais do indivíduo, agindo diretamente no comportamento do mesmo, bem como nas interações sociais, sendo este um processo que ocorre deforma gradativa, pois sabemos que o envelhecimento não surge da mesma maneira para todos (VERAS RP e OLIVEIRA M, 2018).

Mudanças e perdas fazem parte do envelhecimento. A partir dos 40 anos, um indivíduo perde cerca de um centímetro de altura a cada década, principalmente devido à diminuição da altura vertebral devido à osteopenia e outras alterações degenerativas na coluna, a pele fica mais fina, mais frágil, menos elástica e menos oleosa, assim como a visão que também pode diminuir, especialmente com objetos próximos. A audição diminui gradualmente ao longo dos anos, mas geralmente não af eta a vida diária e com a idade, o cérebro perde peso e volume devido à perda de neurônios, mas apesar da redução, a função mental permanece até o fim da vida (JARDIM VCFS, et al., 2019).

Vivemos em uma sociedade cada vez mais excludente em se tratando de idosos, este processo de exclusão é gerado pela sociedade consumista que cada vez mais valoriza o novo, o sagaz as relações e processos imediatos, desta maneira o que não se adapta a este padrão para trás (OLIVEIRA AS, 2019). Várias pesquisas abordam aspectos sobre a velhice e o envelhecer, contudo independente do que se possa retratar acerca desse assunto tão extenso fica sempre algo por descobrir e desenvolver sobre esta temática, uma vez que, o mundo está sempre em constante mudança (MENEZES JNR, et al., 2018).

Desta maneira, surge da necessidade de explorar e tentar compreender que aspectos estão envolto do processo de envelhecimento, dando enfoque à importância do contexto social da idade da velhice, pois de maneira direta indivíduos e sociedade estão envolvidos (JARDIM VCFS, et al., 2019).

O envelhecimento não é uma finalização de uma vida anterior, mas uma continuação da juventude, construção da maturidade, que pode ter sido vivenciada de diferentes maneiras, Além disso, seu contexto histórico em relação à vida privada e pública também desempenha um papel muito importante nos determinantes da velhice (LIMA-COSTA MF, 2018).

O aumento da população idosa no Brasil foi em decorrência do avanço da medicina e também do acesso a saúde, saneamento básico e de higiene, assim como uma alimentação mais saudável e a introdução de atividades físicas na vida dessas pessoas influenciaram nas transformações ocorridas na pirâmide demográfica da população brasileira (VERAS RP e OLIVEIRA M, 2018).

Para refletir sobre o processo de envelhecimento, é necessário principalmente analisar sua relação com a sociedade, uma vez que os sujeitos são constituídos inseridos no meio social ao longo de suas vidas. Afinal, 
cada etapa da vida tem um componente social diferente que influencia os indivíduos a se moldarem de acordo com as transformações que cada etapa do desenvolvimento humano produz. (LIMA-COSTA MF, 2018).

Diante disso, este artigo objetivou analisar como os idosos estão vivenciando o processo de envelhecimento na sociedade.

\section{MÉTODOS}

De acordo com o tema exposto acima, o método de abordagem que utilizou-se na investigação da pesquisa, foi o da Revisão Integrativa da Literatura (RIL), os critérios de inclusão utilizados foram artigos nacionais que tratavam sobre a temática dos idosos em relação ao processo de envelhecimento, em um recorte temporal de cinco anos (2017 a 2021) e que estavam disponíveis no banco de dados dos sites Literatura Latino-Americana e do Caribe em Ciências da Saúde (LILACS), PUBMED e Scientific Electronic Library Online (SCIELO). Foram excluídos trabalhos que necessitassem de taxa de pagamento e escritos em outras línguas.

O primeiro levantamento foi realizado no banco de dados dos sites LILACS, PUBMED e SCIELO, que trouxe um quantitativo de 15 artigos. Após leitura mais apurada desses exemplares e exclusão daqueles que não tratavam da temática selecionada para esta investigação, foi possível eleger e delinear as referências que se faziam imprescindíveis para este estudo. Apesar de serem localizados títulos pertinentes a temática a partir das palavras-chave: velhice, medicamentos, atenção farmacêutica. Uma leitura mais detalhada definiu, em torno de trabalhos principais, constituindo-se assim o corpus deste estudo.

O risco foi mínimo e relacionado a estudos usando técnicas e métodos de pesquisa retrospectiva e estudos sem intervenção ou modificação intencional de variáveis psicológicas e sociais dos idosos que participam entre os quais se consideram: questionários, entrevistas etc., nos quais seja invasivo à intimidade do indivíduo. Acredita-se que o resultado do estudo poderá servir de subsídio para avaliações, quanto à velhice e o importante papel da enfermagem na promoção da qualidade de vida desse público-alvo e fornece uma alta probabilidade de gerar conhecimento para entender, prevenir ou mitigar problemas que afetam o bem estar dos sujeitos de pesquisa e outros indivíduos. A distribuição dos estudos está descrito na Figura 1.

Figura 1 - Distribuição de artigos nas bases de dados.

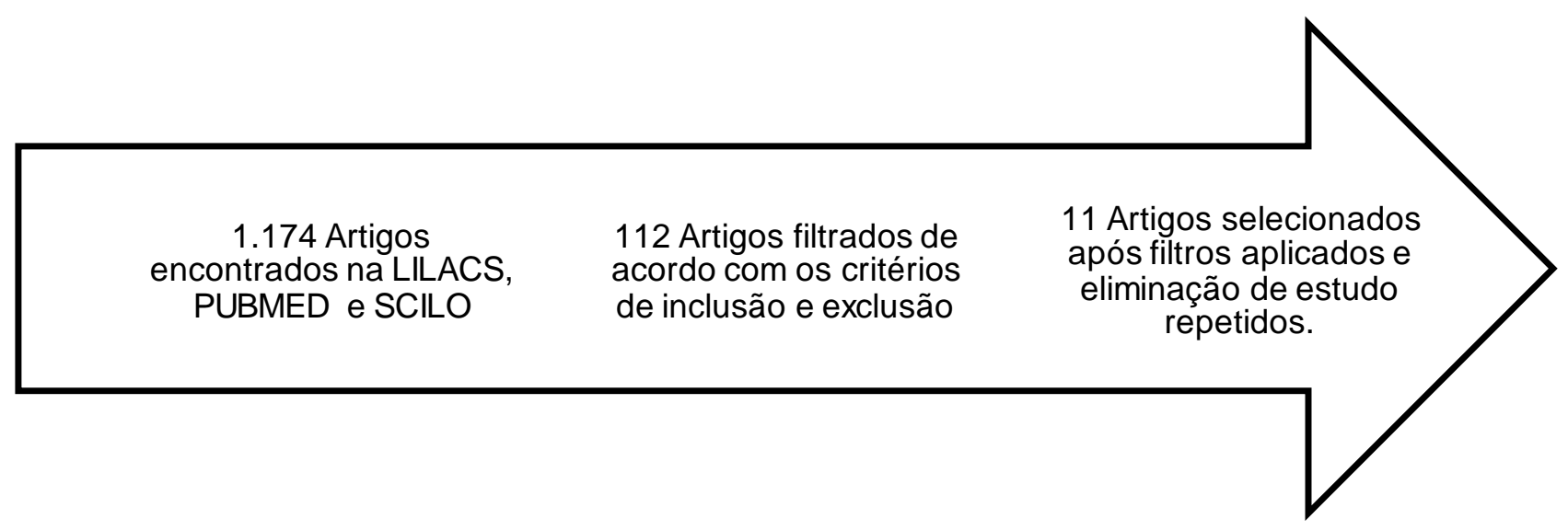

Fonte: De Figueiredo Júnior, et al., 2021

\section{RESULTADOS}

Em uma perspectiva de análise, organizou-se os dados para melhor entendimento, envolvendo autores do periódico, o título dos mesmos e objetivo de estudo que permearam o envelhecimento populacional como descrito no Quadro 1. 
Quadro 1 - Artigos selecionados na Revisão Integrativa da Literatura, 2021.

\begin{tabular}{|c|c|c|}
\hline Autor & Título & Objetivo \\
\hline Myrrha LJD, et al. (2017). & $\begin{array}{c}\text { A contribuição dos } \\
\text { nascimentos e óbitos para o } \\
\text { envelhecimento populacional } \\
\text { no Brasil, } 1950 \text { a } 2010 .\end{array}$ & $\begin{array}{l}\text { Examinar, a partir do método desenvolvido } \\
\text { por Preston, Himes e Eggers (1989), as } \\
\text { componentes responsáveis pelo } \\
\text { envelhecimento da população brasileira, } \\
\text { medido por meio de variações na idade } \\
\text { média populacional ao longo do processo } \\
\text { de transição demográfica, ou seja, entre } \\
1950 \text { e } 2010 \text {. }\end{array}$ \\
\hline $\begin{array}{c}\text { Campos ACV e } \\
\text { Gonçalves LHT (2018). }\end{array}$ & $\begin{array}{c}\text { Perfil demográfico do } \\
\text { envelhecimento nos } \\
\text { municípios do Estado do Pará, } \\
\text { Brasil. }\end{array}$ & $\begin{array}{l}\text { Investigar as diferenças socioeconômicas e } \\
\text { demográficas entre os municípios } \\
\text { paraenses, em relação ao envelhecimento. }\end{array}$ \\
\hline Teixeira SM (2018). & $\begin{array}{l}\text { O Envelhecimento e as } \\
\text { Reformas no Sistema de } \\
\text { Seguridade Social no Brasil } \\
\text { Contemporâneo. }\end{array}$ & $\begin{array}{l}\text { Problematizar os discursos generalizantes } \\
\text { e homogeneizante do envelhecimento } \\
\text { humano, que desconsideram as dif erenças } \\
\text { e desigualdades no modo de envelhecer. }\end{array}$ \\
\hline Alves JED (2019). & $\begin{array}{l}\text { Envelhecimento populacional } \\
\text { no Brasil e no mundo. }\end{array}$ & $\begin{array}{l}\text { Demonstrar o aumento do número absoluto } \\
\text { e do percentual de idosos no conjunto da } \\
\text { população, que ocorre desde 1950, mas, } \\
\text { principalmente, ao longo do século XXI. }\end{array}$ \\
\hline Santos PA, et al. (2019). & $\begin{array}{l}\text { A percepção do idoso sobre a } \\
\text { comunicação no processo de } \\
\text { envelhecimento. }\end{array}$ & $\begin{array}{c}\text { Verificar a percepção dos idosos sobre seu } \\
\text { processo de comunicação no } \\
\text { envelhecimento. }\end{array}$ \\
\hline Pariol CLL, et al. (2019). & $\begin{array}{l}\text { A influência da autoestima no } \\
\text { processo do envelhecimento. }\end{array}$ & $\begin{array}{l}\text { Descrever e discutir as relações entre } \\
\text { influência da autoestima em pessoas } \\
\text { idosas no processo do envelhecimento. }\end{array}$ \\
\hline Caio CB, et al. (2019). & $\begin{array}{l}\text { Envelhecimento e aparência: a } \\
\text { experiência de indianos } \\
\text { imigrantes da cidade de São } \\
\text { Paulo, Brasil. }\end{array}$ & $\begin{array}{l}\text { Apresentar mudanças no curso de vida do } \\
\text { idoso no Brasil. }\end{array}$ \\
\hline Figueira O, et al (2020). & $\begin{array}{l}\text { Estratégias para a promoção } \\
\text { do envelhecimento ativo no } \\
\text { Brasil: uma revisão integrativa. }\end{array}$ & $\begin{array}{l}\text { Analisar como estratégias propostas não } \\
\text { meio científico para uma promoção faz } \\
\text { ativo não cenário brasileiro. }\end{array}$ \\
\hline Queiroz MG, et al (2020). & $\begin{array}{l}\text { Envelhecimento saudável } \\
\text { prejudicado pela obesidade: } \\
\text { uma revisão integrativa. }\end{array}$ & $\begin{array}{l}\text { Descrever e analisar o envelhecimento } \\
\text { humano saudável prejudicado pela } \\
\text { obesidade, af etando a qualidade de vida. }\end{array}$ \\
\hline $\begin{array}{l}\text { Dos santos SEM, et al. } \\
\qquad(2021) .\end{array}$ & $\begin{array}{c}\text { História, conceitos e } \\
\text { concepções sobre o processo } \\
\text { de envelhecimento. }\end{array}$ & $\begin{array}{l}\text { Analisar o surgimento e a construção dos } \\
\text { conceitos de velhice e envelhecimento, } \\
\text { abordando as diversas definições. }\end{array}$ \\
\hline Cepellos V (2021). & $\begin{array}{l}\text { Feminização do } \\
\text { envelhecimento: um fenômeno } \\
\text { multifacetado muito além dos } \\
\text { números. }\end{array}$ & $\begin{array}{c}\text { Estimular o debate sobre o tema no campo } \\
\text { da Administração, a fim de mitigar os } \\
\text { ef eitos de prejuízos advindos da idade. }\end{array}$ \\
\hline
\end{tabular}

Fonte: De Figueiredo Júnior, et al., 2021

\section{DISCUSSÃO}

Observa-se que ainda há uma recusa muito grande por parte do público masculino em relação a sua participação em grupos de terceira idade, compreende- se que essa recusa ocorre por fatores ligados a 
questões de este possuir a cultura de que homem não precis a de inserção nesses espaços para se obter 0 autoconhecimento e realização pessoal (MYRRHA LJD, et al., 2017).

Diante do que já foi discutido no decorrer da pesquisa acerca da temática envelhecimento, percebe-se as distinções que cada idoso tem de como seria envelhecer na atualidade, bem como, as dificuldades que cada um enfrentou ou enfrenta para se chegar a essa etapa da vida (CEPELLOS V, 2021).

Para alguns autores envelhecer é algo maravilhoso que proporciona a chance de corrigir os erros do passado, bem como, amplia o conhecimento acerca do que é a vivência em sociedade, tendo em vista que no passado não possuía essa concepção do que é certo ou errado (ALVES JED, 2019).

Já para outros autores envelhecer é reconhecer os seus direitos de idosos, ainda que este processo de envelhecimento traga consigo as consequências do próprio envelhecer, tais como as dificuldades que o como humano enfrenta nesta fase. Envelhecer é aceitar suas limitações se adequando a realidade de sua família quanto as diferentes faixas etárias existentes em seu convívio e contando com este apoio familiar para enfrentar o processo de envelhecimento (SANTOS PA, et al., 2019).

$O$ fato de se ser idoso(a), não se torna um empecilho para viver a sua velhice de forma limitada, pois envelhecer the permite realizações que antes não pudera realizar enquanto jovem, é sentir-se bem diante de uma sociedade que dita padrões enquanto seu estilo de vida. Envelhecer é não permitir que a sociedade imponha limites para vivermos, pois cada um sabe de suas limitações e todos são capazes de gerir da melhor maneira possível sua vida (PARIOL CLL, et al., 2019).

Fazendo uma comparação com os achados sobre o envelhecimento é perceptível às diversas indiferenças e privações que estes estão sujeitos em seu cotidiano, o que para uns é aceitável suas condições perante a sociedade, para outros se torna incômodo conviver de forma limitada, uma vez que, esse se considera capaz e em plenas condições psíquicas e motoras e a sociedade em si os limita mesmo com a intenção de lhes poupar de esforços físicos, acarretando desta forma sua dependência de outrem (QUEIROZ MG, et al., 2020).

Fica evidente que uma das maiores dificuldades ao vivenciar o processo de envelhecimento ainda está pautada no que tange as Políticas Públicas voltadas ao idoso. Percebe-se, algumas vezes, o descontentamento com o serviço of erecido ao transporte público, ficando evidente o desrespeito à gratuidade que o idoso tem direito (SANTOS SILVA EM, et al., 2021).

Ao analisar pelo olhar da enfermagem os estudos demonstram que o desafio do enfermeiro, O problema de enfrentar os idosos em momentos de exclusão social é promover o diálogo entre as diferentes faixas etárias para despertar a sensibilidade de todos aqueles que sof rem de diferentes formas de discriminação e, ao mesmo tempo, convencer os idosos de que eles têm direitos como seres humanos, que os levará a redescobrir sua verdadeira identidade, assumindo que são eles que são vitais para sua produtividade social (TEIXEIRA SM, 2018).

Atualmente, existem várias evidências empíricas de que os currículos de enfermagem se interessam por conteúdos que enfocam o fenômeno do envelhecimento. Nessa evidência, três se destacam: o aumento gradual da população tornou relevante o interesse pela disciplina de gerontologia; o surgimento de novas necessidades deu ao assunto uma dimensão maior, não apenas biológica, mas agora incluindo também a psicologia do idoso, económica, social e de cidadania; e reconhece o escrutínio das respos tas a estas novas necessidades (CAMPOS ACV, et al., 2018).

Dessa maneira, obtém-se o conhecimento da realidade, bem como o aperfeiçoamento desta temática no meio acadêmico, o que deixaria de ser um simples assunto ministrado para o exercício do futuro pro fissional, torna-se de alguma maneira objeto de conhecimento junto ao qual se insere uma ação transformadora (CAIO CB, et al., 2019).

A enfermagem, Por se tratar de uma profissão em constante interação com políticas e direitos sociais, não pode ignorar o tema do fenômeno do envelhecimento e precisamente este fenômeno que ocorre num ambiente desfavorável à proteção social pública, nomeadamente a regulação econômica e social orientada para o mercado. O desafio para a prática do cuidado ao idoso é conscientizar as pessoas sobre o verdadeiro 
papel das pessoas idosas, garantir seu lugar em uma sociedade que passa por grandes mudanças centradas no avanço tecnológico, em benefício da relação entre mercado e consumo, e a lógica desse, valoriza quem produz e quem consome (FIGUEIRA O, et al., 2020).

Fato de a sociedade capitalista ser útil apenas para o que se produz afeta diretamente a vida e a personalidade dos idosos, que passam a ter alguns comportamentos anormais por se acharem improdutivos, achando que seus comportamentos com os outros são apenas interações com os seus relacionamentos. Convivendo com a natureza através do trabalho. Faz parte da rotina de trabalho da enfermagem, principalmente aqueles inseridos em ambientes de cuidados críticos e de emergência, o processo de envelhecimento, morte e morrer. Este tema, no entanto, não é facilmente resolvido, mesmo os profissionais que, geralmente, reagem diferentemente do evento (MYRRHA LJD, et al., 2017).

Observa-se, na literatura, a insegurança dos profissionais em lidar diretamente com o processo de envelhecimento, morte e morrer, muitos dos quais evitaram mencionaras palavras. Esta constatação está alinhada com os resultados desta pesquisa, na qual se destaca que a resistência em discutir a temática é um dos principais motivos das dificuldades encontrados (CEPELLOS V, 2021).

Sentimentos como impotência, tristeza, frustração e dor são detectados. Pode-se ver que ainda existe um entender que os hospitais têm o objetivo de curar pessoas hospitalizadas e que a velhice e seus possíveis problemas de saúde apresentam-se como uma oposição a isso, os profissionais se percebem como tendo a taref a de lidar com a vida e quando eles testemunham o processo de envelhecimento levando a morte, eles tendem a retratá-la como falha na prestação de cuidados, resultando em sentimentos como angústia, culpa, estresse e impotência (ALVES JED, 2019).

A experiência profissional é uma ótima aliada para lidar com estas questões. A segurança obtida pela execução exaustiva da prática da assistência dos múltiplos casos clínicos comprovados com fins variados mostra-se relevante, em estudos que profissionais de saúde, especialmente enfermeiros, que trabalham com o processo de envelhecimento no trabalho diário é desafiador, mas em muitos casos eles podem reconhecer que é uma vida natural, e que, inevitavelmente, todo mundo irá um dia através dela e isso faz parte da realidade diária de trabalho. Tenta- se criar estratégias para lidar com o processo de envelhecimento e possivelmente com posterior morte com o mínimo de emoção e envolvimento porque, mesmo que não exista reação terapêutica por parte do paciente, sente-se realização (SANTOS PA, et al., 2019).

Diante do exposto, acredita-se que os resultados desta pesquisa podem af etar a prática e ensino. Salientase que atividades educativas para lidar com o processo de envelhecimento, morte em serviços de saúde, especialmente aqueles que oferecem atendimento crítico e de emergência a pacientes em diferentes ciclos da vida, são estratégias que devem ser utilizadas pelos serviços de educação continuada das unidades, assim como universidades/faculdades (CEPELLOS V, 2021).

É possível, portanto, que os profissionais se sintam de alguma forma, mais bem preparados para o enfrentamento diário do processo de envelhecimento e com o paciente na finitude da vida e de sua família. Considera-se que os resultados deste estudo contribuirão para o avanço do conhecimento na medida em que demonstrem a deficiência da abordagem da pessoa em processo de envelhecimento e estimule a capacit ação de profissionais das áreas (CAIO CB, et al., 2019).

Cabe a enfermagem, o fortalecimento das suas funções educativas e políticas, dedicadas aos direitos sociais dos idosos, a salvaguardar a sua dignidade, estimular o seu sentido de participação, integrar-se com as pessoas, trabalhar com os idosos na sua particularidade e singularidade, tendo em conta a sua um todo complexo e contraditório, assim como valorizar sua individualidade e representatividade no contexto social como ser humano único e participativo, assim é possível criar uma sociedade mais justa quando o assunto é direitos humanos da população idosa quebrando preconceitos e estigmas (ALVES JED, 2019).

No que tange a família e a sociedade civil, o enfermeiro em sua principal missão é pensar e refletir sobre as questões do idoso a partir de uma visão transformadora e crítica, despertando o cuidado e o respeito ao idoso. Isso servirá como uma demonstração de respeito pelo nosso próprio futuro à medida que envelhecemos e inevitavelmente enfrentamos a velhice. Dessa maneira, entende-se que os significados que 
cada sujeito tem a despeito da velhice e do envelhecimento, demonstra a heterogeneidade que cada idoso tem de vivenciar a velhice, bem como demonstra a necessidade de se ter um olhar diferenciado para lhe dar com esta etapa mais avançada da vida (CEPELLOS V, 2021).

\section{CONSIDERAÇÕES FINAIS}

A realização desta pesquisa demonstrou que de modo geral, os idosos tem a percepção do processo do envelhecimento de forma positiva, descartando a ideia da imagem defasada do envelhecimento e o estereótipo do idoso triste, dependente, doente e incapacitado, onde a velhice é vista como uma fase complementar ao processo de existência do ser humano. Entretanto, observou-se como ponto negativo, não o processo de envelhecimento em si, mas o descaso por parte do poder público em proporcionar políticas públicas eficazes que atendam a população idosa de forma digna e respeitosa. Analisar o processo de envelhecimento engloba diversos aspectos correlacionados e dependentes entre si, dessa maneira não devem ser abordados de forma isolada. É notável que a temática envelhecimento passou a ter mais visibilidade em torno de estudos e pesquisas cientificas, contudo o que se observa no cotidiano é que este processo ocorre de forma incompatível na pratica e na maioria das vezes não condiz com o que é proposto na legislação que ampara o direito e garantia dos idosos.

\section{REFERÊNCIAS}

1. ALVES JED. Envelhecimento populacional no Brasil e no mundo. Revista Longeviver, 2019; I(3).

2. CAIO CB, et al. Envelhecimento e aparência: a experiência de indianos imigrantes da cidade de São Paulo, Brasil. Revista Kairós: Gerontologia, 2019;22(26):101-125.

3. CAMPOS ACV, et al. Perfil demográfico do envelhecimento nos municípios do Estado do Pará, Brasil. Revista Brasileira de Enfermagem, 2018;71:591-598.

4. CEPELLOS V. Feminização do envelhecimento: um fenômeno multifacetado muito além dos números. Revista de Administração de Empresas, 2021;61.

5. DOS SANTOS SILVA EM, et al. Envelhecer na Periferia. História, conceitos e concepções sobre o processo de envelhecimento. Revista Longeviver, 2021; III(9).

6. FIGUEIRA O, et al. Estratégias para a promoção do envelhecimento ativo no Brasil: uma revisão integrativa. Research, Society and Development, 2020;9(10).

7. JARDIM VCFS, et al. Um olhar sobre o processo do envelhecimento: a percepção de idosos sobre a velhice. Revista brasileira de geriatria e gerontologia, 2019; (9): 25-34.

8. LIMA-COSTA MF. Envelhecimento e saúde coletiva: estudo longitudinal da saúde dos idosos brasileiros (ELSI-Brasil). Revista de Saúde Pública, 2018; (52).

9. MENEZES JNR, et al. A visão do idoso sobre o seu processo de envelhecimento. Revista Contexto \& Saúde, 2018; 18(35):8-12.

10. MYRRHA LJD, et al. A contribuição dos nascimentos e óbitos para o envelhecimento populacional no Brasil, 1950 a 2100. Revista Latino-americana de Población, 2017;11(20):37-54.

11. OLIVEIRA AS. Transição demográfica, transição epidemiológica e envelhecimento populacional no Brasil. HygeiaRevista Brasileira de Geografia Médica e da Saúde, 2019;15(32):69-79.

12. PARIOL CLL, et al. A influência da autoestima no processo do envelhecimento. Diálogos Interdisciplinares, 2019; 8(1): 45-52.

13. QUEIROZ MG, et al. Envelhecimento saudável prejudicado pela obesidade: umarevisão integrativa. Brazilian Joumal of Health Review, 2020; 3(2): 2309-2316.

14. SANTOS PA, et al. A percepção do idoso sobre a comunicação no processo de envelhecimento. AudiologyCommunication Research, 2019;24.

15. TEIXEIRA SM. O Envelhecimento e as Reformas no Sistema de Seguridade Social no Brasil Contemporâneo. Textos \& Contextos (Porto Alegre), 2018; 17(1):126-137.

16. VERAS RP, OLIVEIRA M. Envelhecer no Brasil: a construção de um modelo de cuidado. Ciência \& saúde coletiva, 2018;23: 1929-1936. 